\title{
PLANT CONDITION REMOTE MONITORING TECHNIQUE
}

\section{Conf pare}

\author{
L.K. Fotedar \\ University of Texas- Health Science Center at Houston \\ Suite 7.044 \\ 6431 Fannin Street \\ Houston, TX 77030 \\ K. Krishen, Ph.D. \\ Technology Transfer and Commercialization Office \\ Code HA111 \\ NASA Johnson Space Center, Houston, Texas 77058
}

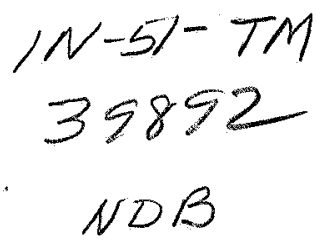

\begin{abstract}
This paper summarizes the results of a radiation transfer study conducted on houseplants using controlled environmental conditions. These conditions included: (1) air and soil temperature; (2) incident and reflected radiation; and (3) soil moisture.
\end{abstract}

The reflectance, transmittance, and emittance measurements were conducted in six spectral bands: microwave, red, yellow, green, violet, and infrared, over a period of three years. Measurements were taken on both healthy and diseased plants.

The data was collected on plants under various conditions which included: variation in plant biomass, diurnal variation, changes in plant pathological conditions (including changes in water content), different plant types, various disease types, and incident light wavelength or color. Analysis of this data was performed to yield an algorithm for plant disease from the remotely sensed data.

\section{INTRODUCTION}

One key application of the space technology developed by the National Aeronautics and space Administration (NASA) has been in the area of global monitoring of earth resources (Ref.1). In cooperation with the United States Department of Agriculture (USDA), NASA has established the potential for remote sensing through the Landsat program.

This program is concerned with the estimation of crop yield from infrared and visible imagery. More recently, several organizations throughout the world are investigating the potential of microwave systems to complement the Landsat sensors for all weather predictions. Numerous publications on the subject of remote sensing of crops identify physical variables, such as: crop type, maturity/age, soil moisture, farming practices, crop health, and climatic factors, as significant contributors to the crop "signature" (Ref. 2). A mathematical model (based on both microwave and infrared data) which could separate these variables has, to our knowledge, not yet emerged.

The present study was undertaken to isolate and study the effect of plant condition/health on the reflectance/emittance in six spectral bands: microwave, red, yellow, green, violet, and thermal infrared.

Disease in plants is brought about by infection with fungi, bacteria, viruses, nematodes, algae, and parasitic higher plants (Ref. 3). Deficiency of chemicals such as iron, nitrogen, calcium, and water may also cause disease. In this project, plants with rust, smut, leaf spot, virus, and mineral deficiency diseases were studied (Ref 4).

Incident radiation on the plant undergoes three processes for each of the three parts of the electromagnetic spectrum: reflection, radiation emission, and transmission (Ref. 5). These three processes have a bearing on the plant's efficiency in the use of radiation and its adaptability for survival under stress conditions (disease) (Ref 6,7).

Different methods of plant disease detection have been incorporated. One method is by viewing leaf, stem, or root cross-sections under a microscope or more complex optical source (Ref. 3,6). Another method is just by visual observation of outward symptoms of the disease. Biochemical analysis of the plant biomass is also used (Ref. 8,9). All these methods, however, employ invasive and often destructive measures to detect diseases and are not 
economical or even accurate for large areas/volumes of vegetation.

The key element of this investigation is that measurements were taken while the plant was living in its full biomass (in vivo), and undergoing normal energy processes.

Houseplants were chosen so that plants with slow and fast growth could be used in the present study. Any changes in the plant condition caused by disease or lack of moisture cause changes in the energy transfer processes (evapotranspiration, conduction, and convection) (Ref. 10): For this reason, the radiated or reflected energy in the visible, infrared, and microwave bands is different for diseased plants. Reflected and/or emitted energy provides an indication of the health of the plant.

Measurements were taken on both healthy and diseased plants under laboratory type conditions. The data was collected under various conditions which included: plant biomass, diurnal variation, surrounding temperature, amount of light, pathological conditions (water content, frozen/unfrozen, algae, etc.) and incident lighting conditions.

Establishing repeatability of the data was a key concern and was addressed throughout the study. For this reason, a controlled environment (room) was used to observe six types of houseplants. The added advantage of controlled environment was that the plant could be constantly observed under unperturbed conditions with full biomass and through a phase/history of diseases which interfered with its normal energy exchange processes. The plants were accessible day and night for these observations.

The data shows that water deficiency in the plant, and the plant biomass have strong dependence in the microwave band. The diseased plants show measurable difference compared to healthy plants in the reflected visible and emitted infrared bands. A mathematical formula was proposed on the basis of the observed dependencies for monitoring the plant condition. This algorithm is now ready for testing/evaluation under natural conditions.

\section{INSTRUMENTS AND PROCEDURE}

The development of the expression for the plant condition remote monitoring technique was based on the normalized integrated measurements of plant reflections/emissions at six wavelengths over a period of two years. The wavelengths used were $0.41,0.45,0.55,0.65$, and 11 microns, and $3 \mathrm{~cm}$. A PRT-5 radiometer and thermiscope were used at the 11 micron band.

The radiometer gives point measurements of the temperature on the leaves. The thermiscope showed temperature differences of the objects in imagery form and pictures were taken for record and analysis. The microwave system consisting of an antenna, transmitter, receiver, oscilloscope, amplifier, and a power meter, was used for the $3 \mathrm{~cm}$ band.

In addition to these instruments, an accurate thermometer, a light exposure meter, and microscope were used to characterize the light environment and the plant status.

A controlled environment (room) was made ready for some experiments by placing black cloth around the plant to absorb background radiation. The room temperatures were maintained to within 1 degree centigrade for the periods of observation.

Several experiments were conducted in the patio under uncontrolled environmental conditions. Soil moisture data was also collected in addition to temperature and lighting data. Figures 1, 2, 3 illustrate typical instrument block diagrams and setup.

The plants were rotated to cover all the foliage for the reflectance, transmittance and emissivity. An average was computed for each plant.

Six types of plants ( the Crassula Philodendron Ficus, Shefflera Tradescantia and Vriesea) were selected with four types of diseases (rust, fungus, virus, and deficiency). Data in the five spectral bands was taken daily with the following variables: (1) plant biomass; (2) diurnal variation (morning, afternoon, and night); (3) plant type; (4) disease type; (5) room temperature; and (6) incident radiation.

The $3 \mathrm{~cm}$ wavelength was found very sensitive to the water content of the plant (actual weight minus dry weight). Transmittance and reflectance measurements were taken for several plants in this band, and the plant was rotated to obtain an average value for the plant.

Plots and tables for the average values and variances were computed along with a record of the plant biomass and stage of growth. 


\section{DATA COLLECTED}

Several types of data were taken to account for the different variables involved in the experimentation of this technique. Figure 4 shows the marked difference in the band readings between a diseased Schefflera plant and a healthy one. The healthy plant has a higher reflectance reading in the green, yellow, and red bands, a higher emission reading in the infrared, and a lower transmittance reading in the $x$-band (since the healthy plant has more foliage).

In Figure 5, different diseases were compared. The two diseases compared were nitrogen deficiency and rust fungus. The deficiency disease had a higher reading in the infrared band, and, again, a lower reading in the $x$-band. Additional data collected consisted of a photo image of the plant vs. the thermiscope image. In one case for the Philodendron, the disease was shown on the infrared scan two weeks before it showed up on the plant.

Figure 6 shows the diurnal variation between a healthy Tradescantia plant and a diseased one. Both plants have the highest emittance readings in the afternoon, and the lowest in the morning. Figure 7 shows the difference in microwave transmittance readings of Ficus and Palm plants. Specific weather conditions are tested in this experiment. The Palm plant (having frostbite) showed a considerably higher transmittance than the deficiency Ficus. In Figure 8, plant biomass was tested.

Data taken in all five bands showed the larger Vriesea plant to have higher values than the smaller plant. The difference was especially noticeable in the X-band. Each of these samples of data showed proof of the basic hypothesis first presented.

\section{DETECTION TECHNIQUE}

The repeatability of the measurements was ascertained to within $1 \%$. The dependencies in the graphs indicate that the water deficiency in plants can be detected most accurately in the microwave band.

The diseased plants show measurable differences compared to healthy plants in the reflected visible and emitted infrared bands. The parasitic and noparasitic diseases of plants show differences in all the spectral bands investigated. Diurnal variation readings show that plant emittance is at its highest during the afternoon.

Based on the graphs presented, a detection technique for the plant condition is proposed. The responses (relative emittance, reflectance, and transmittance) in the visible, infrared, and microwave bands are labeled as $A_{i}$ 's. These responses were measured for both healthy $\left(A_{i}{ }^{\prime} s\right)$ and diseased $\left(B_{i}^{\prime}\right.$ 's) plants of the same type. Ratios $B_{i} / A_{i}$ were computed for the known plant diseases.

Based on these measurements, a quantity

$\mathrm{K}=\sum_{i} \mathrm{~W}_{\mathrm{i}}\left(\mathrm{B}_{\mathrm{i}} / \mathrm{A}_{\mathrm{i}}\right)$

where $W_{i}$ are weighting factors for

various bands that was computed to emphasize the use of a particular band for a specific disease. A matrix of $W_{i}$ 's and $X_{i}$ 's for various plant types, stages of growth, diseases, and environmental factors was developed under known conditions. The algorithm showed more than $96 \%$ correlation between the observed plant condition and the predicted disease/water deficiency.

\section{v. CONCLUSIONS}

In a particular application, data should be collected over known healthy vegetation ( $A_{i}$ 's) using handheld, vehicle-mounted, aircraft, or spacecraft data. Data should also be collected under unknown conditions and labeled as $B_{i}$ 's.

The value of $K$ should be computed and compared with the memorized values in the computer. The memorized values are developed using known plant condition and laboratory observations. These values can be experimentally determined using controlled environments similar to what was done in this study. The comparison would then yield the unknown vegetation disease/condition.

The procedure was tested using the data presented in Section III. It was found to be accurate nine times out of ten for the diseases/conditions and plants for which the data was collected. The procedure could be applied to the commercially available Landsat data for large area applications (Ref.11).

In conclusion, this study successfully identifies a quantitative noninvasive remote monitoring technique to detect pathological or water deficiency related conditions in living plant based on significant and sensitive variation in their unique electromagnetic spectral signatures. 


\section{ACKNOWLEDGMENT}

The authors wish to acknowledge the comments and suggestions given by Mr. Jerry Elliott, High Eagle, of NASA Johnson Space Center.

\section{REFERENCES}

1. Sheffner, E. J., The Landsat Program: Recent History and Prospects, Photogrammetric Engineering and Remote Sensing, 60: 735-744, 1994.

2. Erickson, John D., Satellite-Aided Global Crop Monitoring, Microwave Remote Sensing Symposium proceedings, sponsored by National Aeronautics and Space Administration, Johnson Space Center, December, 1977.

3. Devling, Robert M., Plant Physiology, New York; Reinhold Publishing Corp., 1966.

4. Corbett. m. Plant Virology, University of Florida, Florida, 1967.

5. King C., "Agricultural Terrain Scatterometer Observations with Emphasis On Soil Moisture Variation", University of Kansas, Tech. Rept. RSL 177-44, Kansas, 1973.

6. Slayter, R.O., Plant Water Relationships, Academic Press, London, 1967.

7. Ashby, Maurice, Introduction to Plant Ecology, St. Martin's Press, New York, 1969.

8. Grodzinskii, D.N., Plant Biophysics, Jerusalem: Keterpress Enterprises, Inc., 1976.

9. Bickford, E.D., and S. Dunn, Lighting for Plant Growth, Kent state University Press, Inc., 1972.

10. Ulaby, F.T., R.Y. Li, and K.S. Shanmugam, "Crop Classification Using Airborne Radar and Landsat Data", university of Kansas, Kansas, Issued by NASA as SA-KI-04043 Report, Feb., 1981.

11. Salomonson, V.V., J.R Irons, and D.L. Williams, The Future of Landsat: Implications for Commercial Development, Proceedings of the Conference on NASA Centers for Commercial Development of Space (NASA CCDS), M.S. El-
Genk and R.P. Whitten (eds.), American Institute of Physics, New York, 353-359, 1995. 


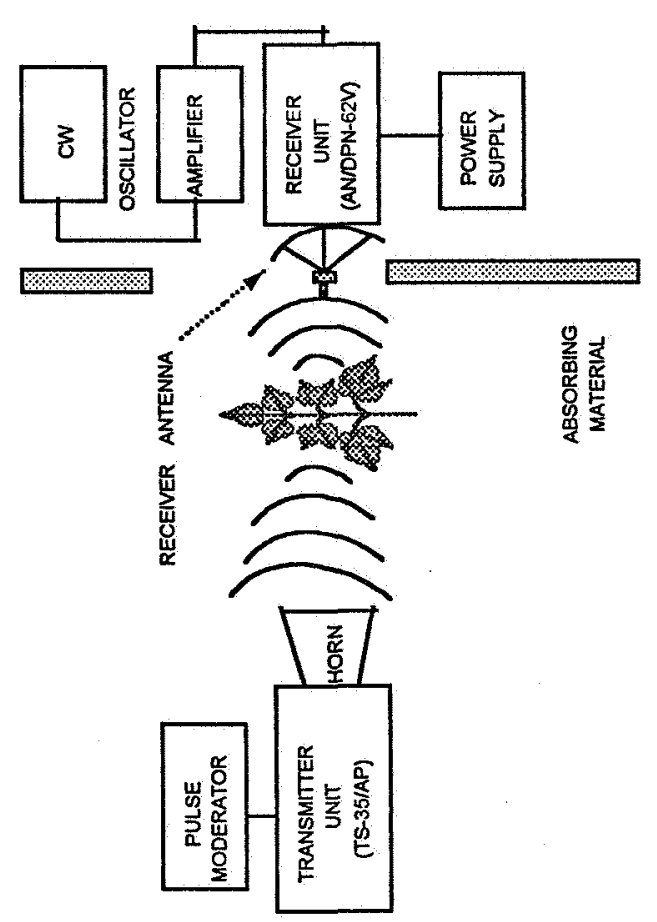

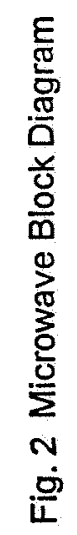
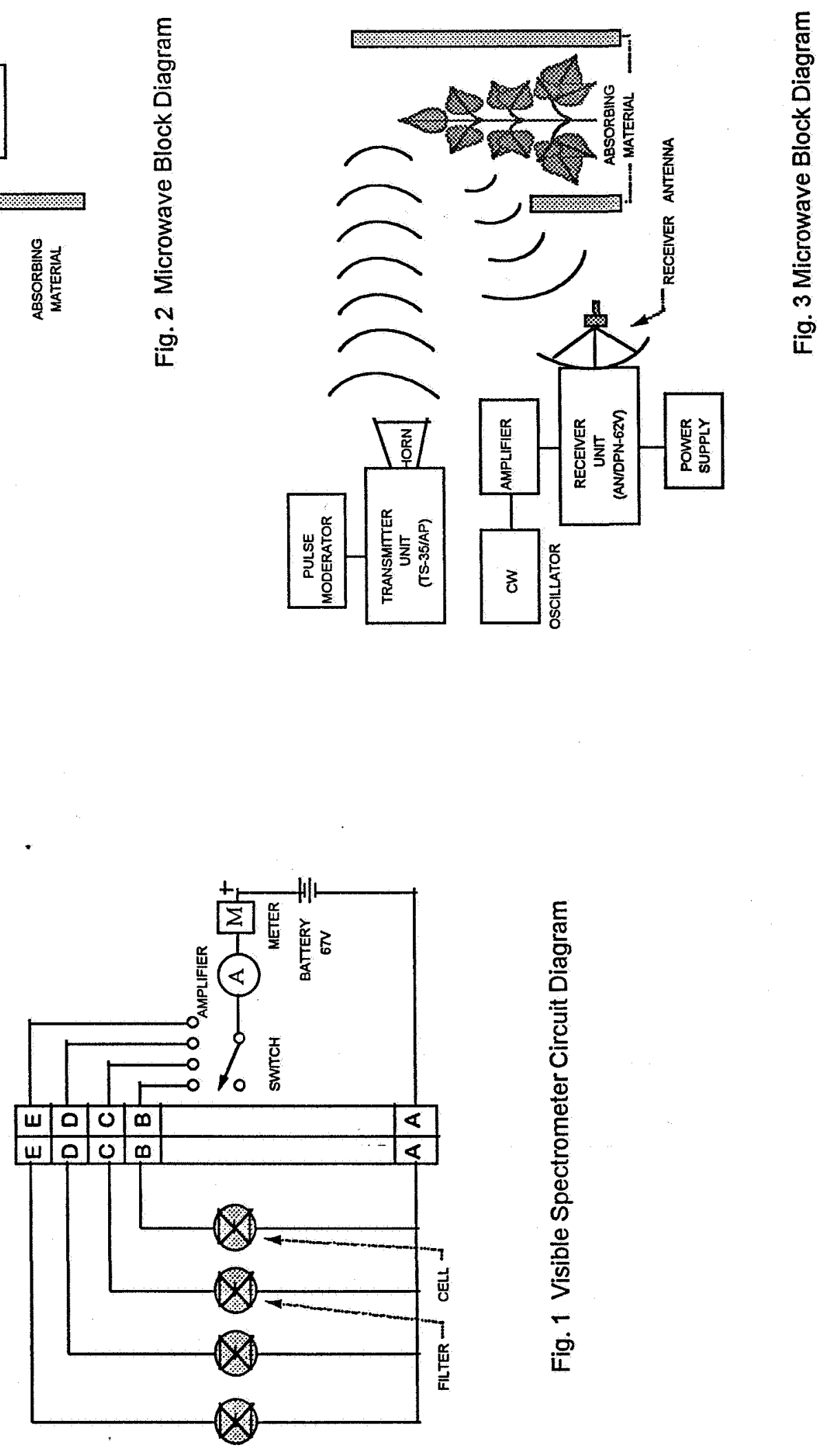


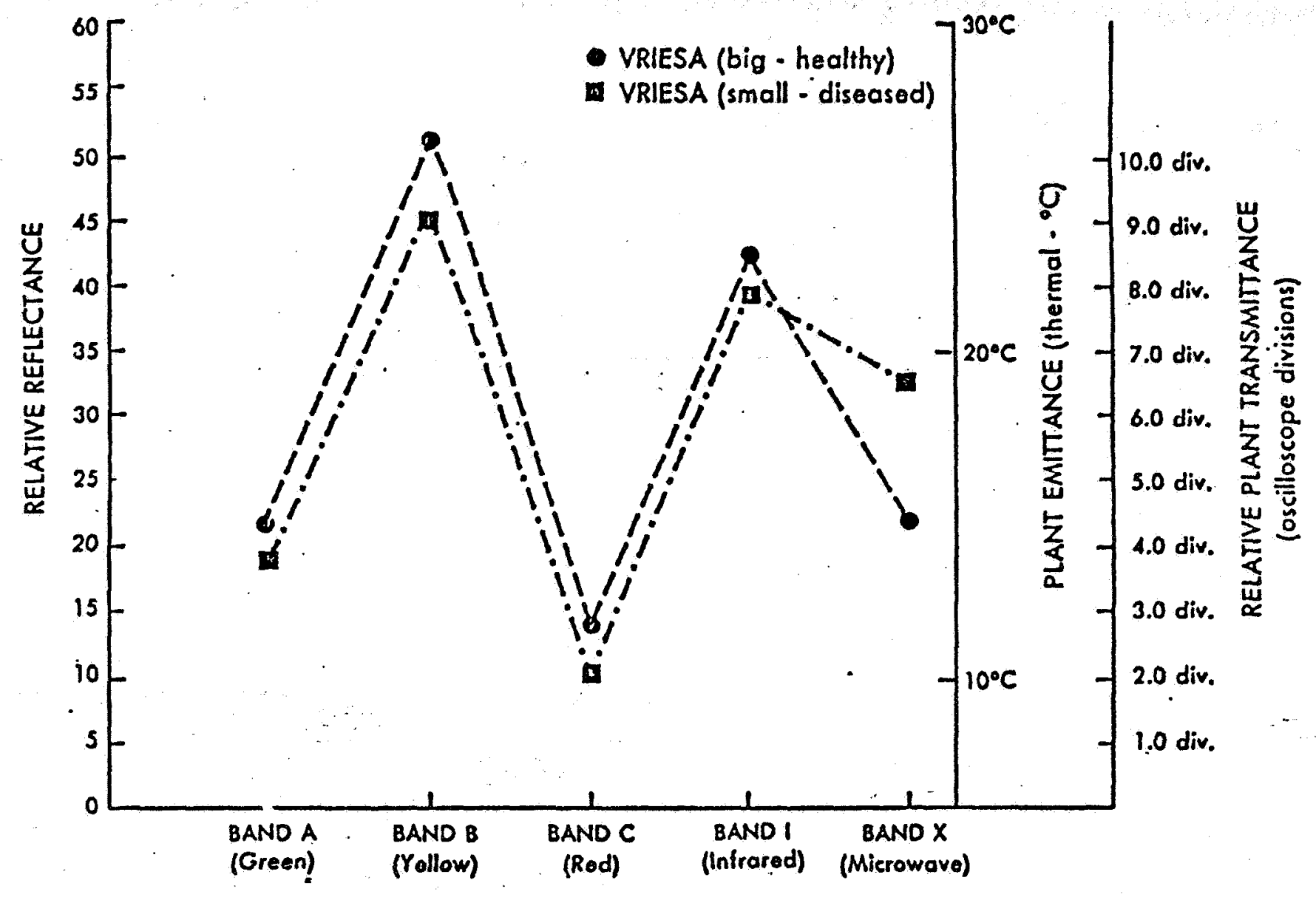

Fig. 8 Plant Biomass 


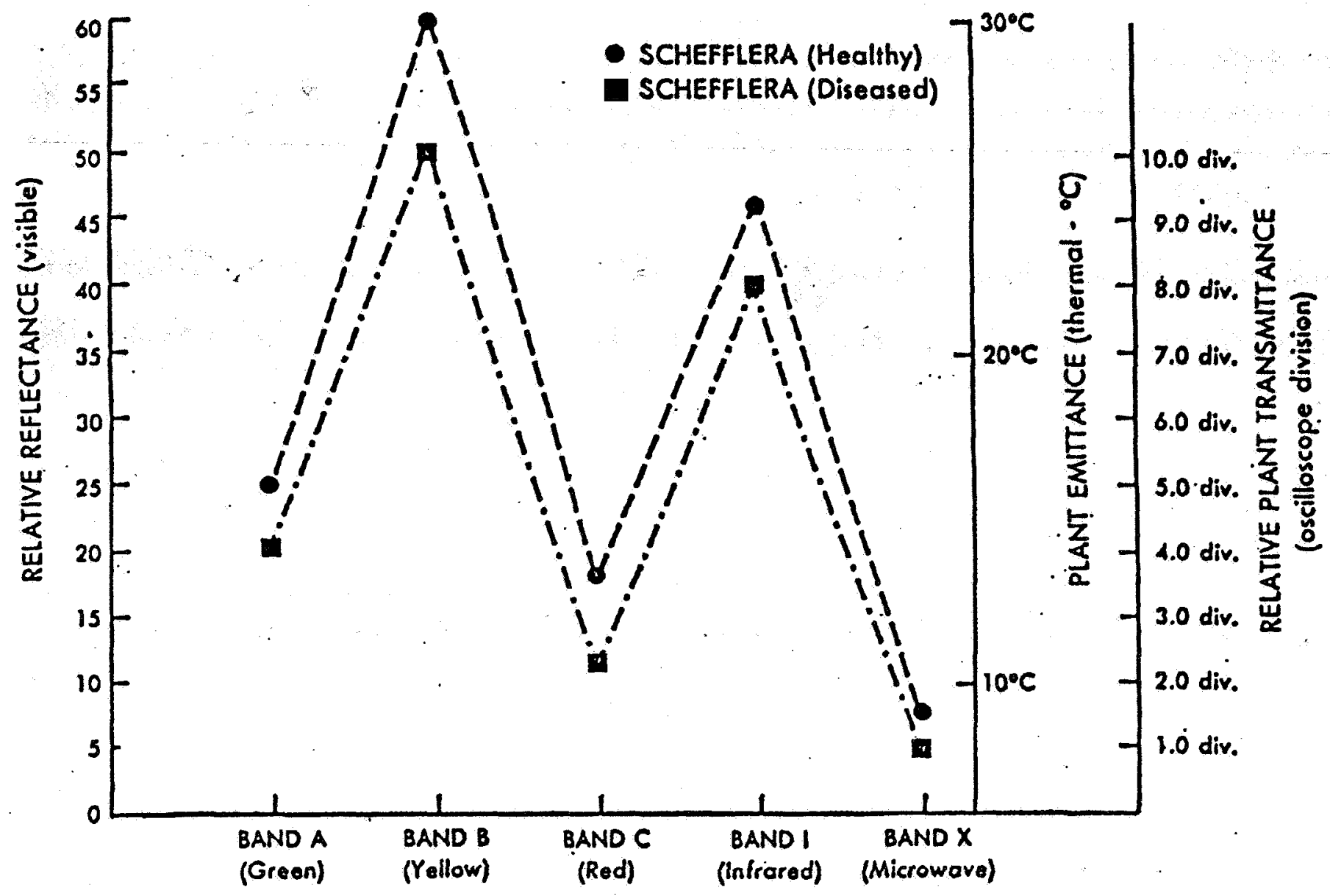

Fig. 4 Healthy Vs. Deseased Plant

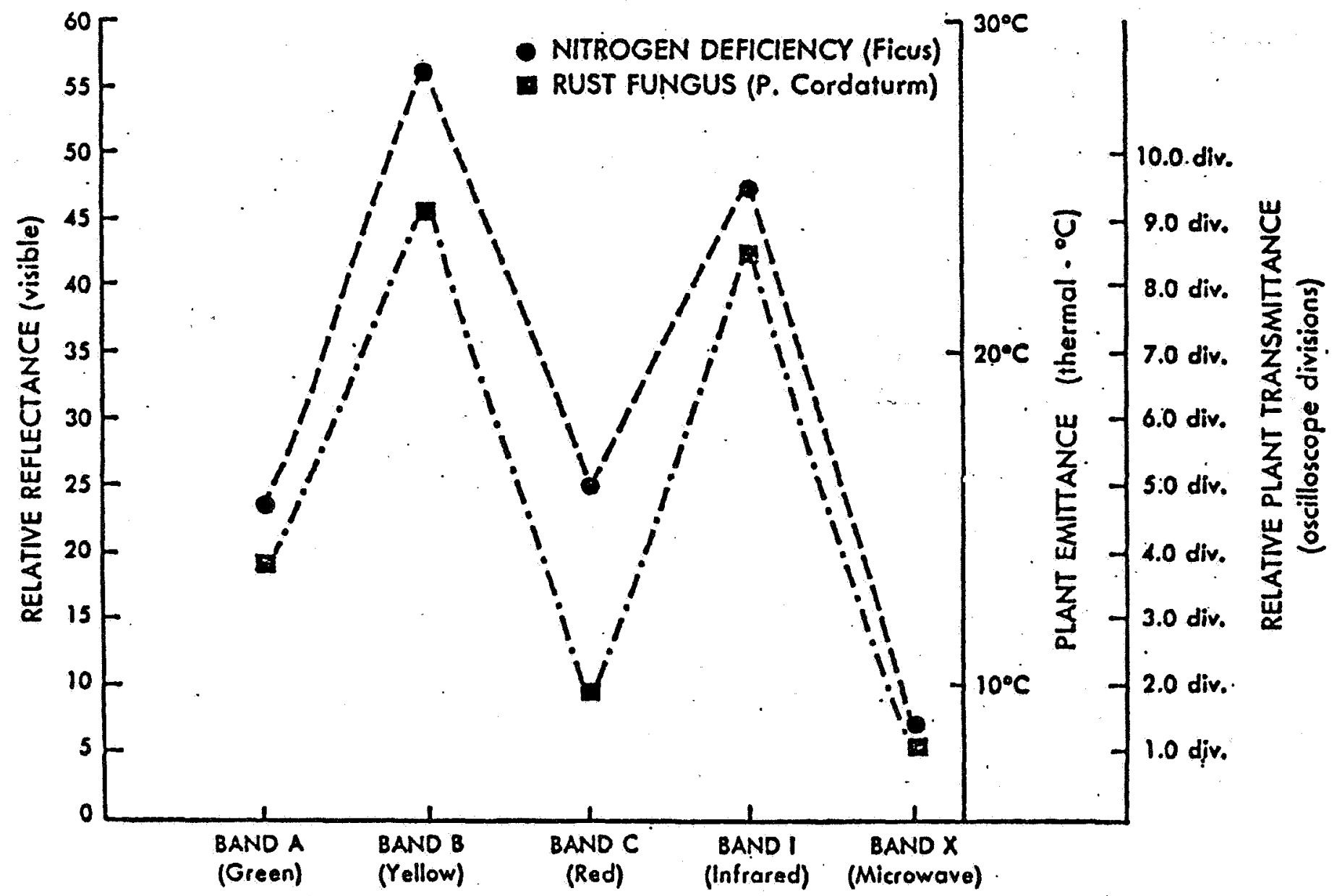

Fig. 5 Response With Disease Types 


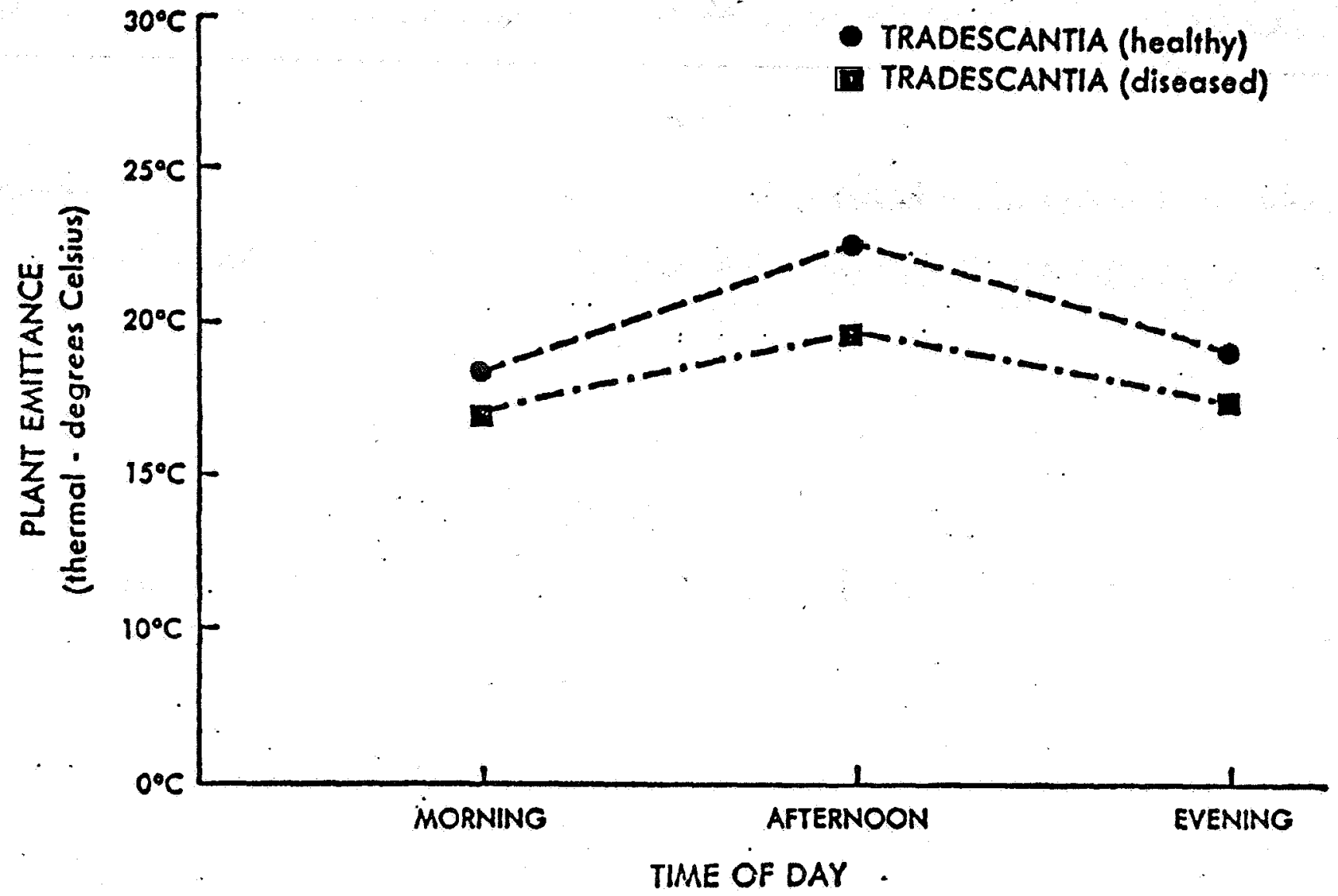

Fig. 6 Diurnal Variation (Average Values)

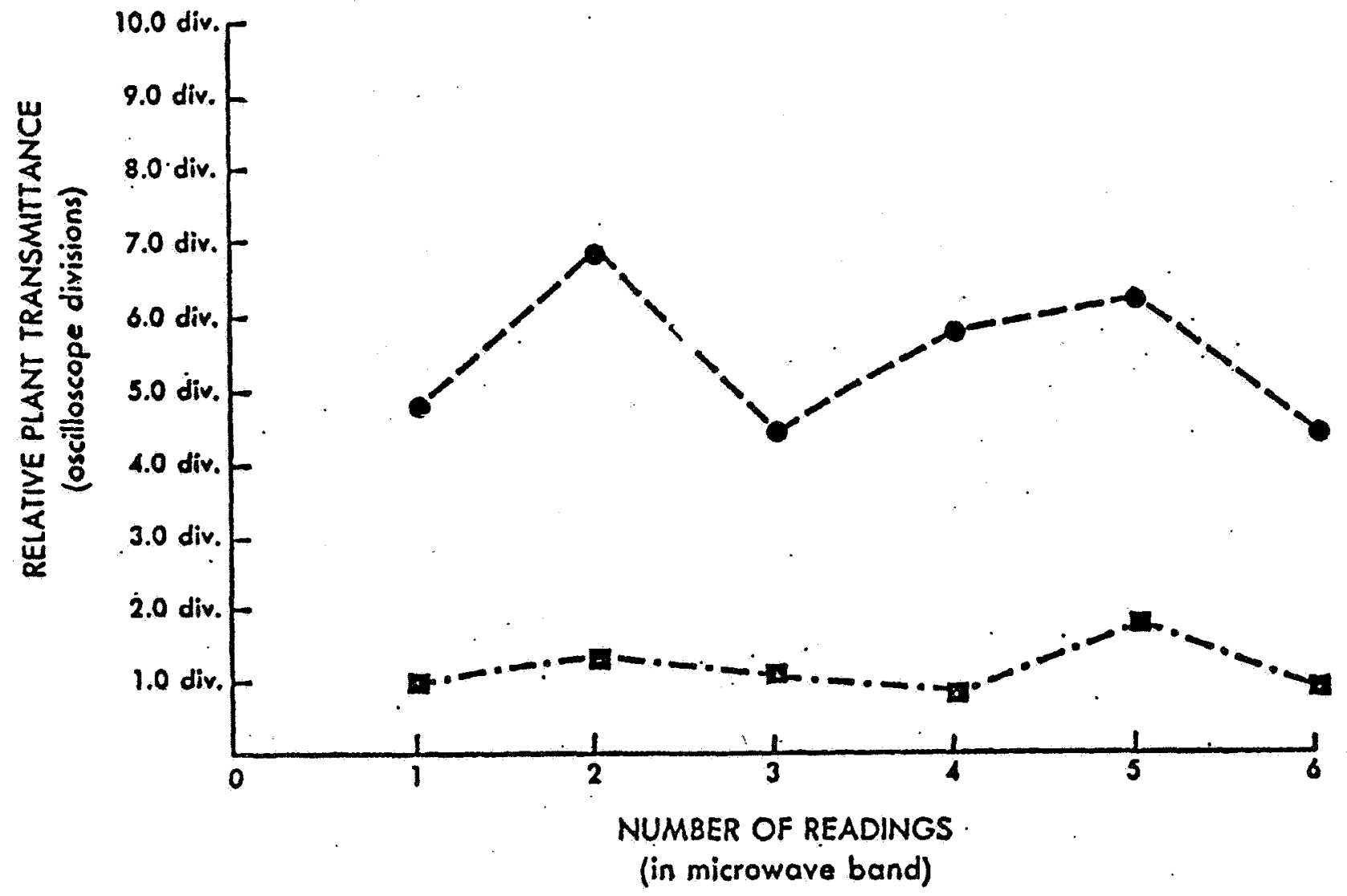

Fig. 7 Ficus (Nitrogen Deficiency) Vs Palm (Frostbite) 